\title{
A Liability Tracking Portfolio for Pension Fund Management
}

\author{
Masashi Ieda $^{\dagger *}$, Takashi Yamashita ${ }^{\ddagger}$ and Yumiharu Nakano ${ }^{\dagger}$ \\ ${ }^{\dagger}$ Graduate School of Innovation Management, Tokyo Institute of Technology \\ 2-12-1 Ookayama, Meguro-ku, Tokyo, Japan \\ *The Government Pension Investment Fund, Japan \\ 1-4-1 Kasumigaseki, Chiyoda-ku, Tokyo Japan \\ E-mail: ieda@craft.titech.ac.jp*
}

\begin{abstract}
We study the long term portfolio which is able to track a liability. The portfolio optimization problem is defined as the stochastic optimal control problem and the performance criterion is the lower mean square error between the liability and our wealth. We impose constraints for the portfolio weights and obtain the optimal portfolio strategy numerically by solving the Hamilton-Jacobi-Bellman equation applying the quadratic approximation scheme. The numerical simulations using the empirical data provided by Japanese organizations are run under the two types of constraints: the no-short-selling constraint; the upper bound constraint for the portfolio weights. The former demonstrates that the liability tracking ability of our optimal portfolio strategy does not drop if we restrict the short selling. The latter implies the imbalance between the growth rate of the liability and the profitability of the assets.
\end{abstract}

\section{Introduction}

A portfolio strategy taking into account the liability is one of the significant issues in the pension fund management. Due to the population ageing especially observed in the developed countries, it is expected that the benefit of the welfare pension fund increases and his or her contribution decreases. Moreover the low birth rate implies that this relation is not resolved for decades. Thus the pension funds face a problem to include the method for hedging their liabilities into their long term portfolio management.

In contrast, a lot of practitioners in pension funds determine their portfolio by the traditional single period mean variance approach. It is difficult to adopt this approach to include a view of hedging the liability since the single time period method is unable to allow us to change the portfolio after the initial time. Although the multi time period method allows the change after the initial time, we face a problem of the computational cost: we are usually unable to obtain the optimal long term portfolio strategy in realistic time.

The literature [1] studies the long term pension fund management taking into account the liability employing the LQG (Linear, Quadratic cost, Gaussian) control problem which is a class of stochastic control problem. Since the analytical solution of the LQG control problem is available, the optimal portfolio strategy is obtained in realistic time.
It demonstrates that the optimal portfolio strategy tracks the liability with the low tracking error by the numerical simulation using the empirical data provided by the Japanese organizations.

In the present paper, we develop the portfolio strategy from the strategy proposed by Ieda et. al. [1] mentioned above. The previous study remains two points which should be improved: (i) penalising the wealth of the investor exceed the liability; (ii) permitting the large short selling. To resolve these points, we employ a lower mean square error from the tracked liability as the performance criterion and restrict the portfolio weight to a positive and bounded value. In this case we are not able to obtain an analytical solution and thus we employ the numerical method to solve the corresponding Hamilton-Jacobi-Bellman (HJB) equation. Since we have currently consider the portfolio constructed by multi assets which correlate each other, it is difficult to obtain a solution in general: for instance, the finite difference method is failed in this case (see e.g., Kushner and Dupuis [2]). To cope with this problem, we employ the quadratic approximation scheme proposed by Nakano [3]. The advantage of this method is the low computational cost, and hence applying this method, we are able to obtain a proxy of the optimal portfolio strategy in realistic time.

Main implications from the present work are as follows. The first remarkable one is that the liability tracking ability of our optimal portfolio strategy does not drop if we restrict the short selling. The mean hedging error is approximately $4.5 \%$ of the liability and it is the equivalent level without the no-short-selling constraint studied in [1]. The hedge is realized by borrowing the money a lot and investing the lowrisk assets. We next note that restricting the large money borrowing by imposing the upper bound constraint for the portfolio weights, we find a number of sample paths which have a large tracking error. It implies that the imbalance between the growth rate of the liability and the profitability of the assets.

The present paper is organized as follows. Section 2 gives the mathematical formulation of the problem. The processes describing the assets and the liability is defined as the stochastic differential equations, and we adopt the lower mean square error between the liability and our wealth as the performance criterion. The numerical method is presented in Section 3. We describe the procedure to obtain the optimal portfolio strategy according to the quadratic ap- 
proximation scheme with regression method. In Section 4, numerical simulations using the empirical data provided by the Japanese organizations are served. We regard the shortfall of income of the pension fund as the liability and hedge it under the following two types of constraints: (i) the noshort-selling constraint in Section 4.1; (ii) the upper bound constraint for the portfolio weights in Section 4.2.

\section{Model}

Let $\left(\Omega, \mathcal{F},\left\{\mathcal{F}_{t}\right\}_{t \geq 0}, \mathbb{P}\right)$ be a filtered probability space and let $\left\{W_{t}\right\}_{t>0}$ be a $n$-dimensional Brownian motion. We denote by $S_{t}^{0}, S_{t}=\left(S_{t}^{1}, \cdots, S_{t}^{n}\right)^{\top}$ and $Y_{t}$ prices of a risk-free asset, risky assets and a liability at time $t$ which are governed by the following stochastic difference equations:

$$
\begin{aligned}
& \left\{\begin{array} { l } 
{ \frac { d S _ { t } ^ { 0 } } { S _ { t } ^ { 0 } } = r ( t ) d t , } \\
{ S _ { 0 } ^ { 0 } = s _ { 0 } ^ { 0 } > 0 , }
\end{array} \quad \left\{\begin{array}{l}
\frac{d S_{t}^{i}}{S_{t}^{i}}=b^{i}(t) d t+\sum_{j=1}^{n} \sigma^{i j}(t) d W_{t}^{j}, \\
S_{0}^{i}=s_{0}^{i}>0, \quad i=1,2, \cdots, n,
\end{array}\right.\right. \\
& \left\{\begin{array}{l}
d Y_{t}=\left(A(t) Y_{t}+B(t)\right) d t, \\
Y_{0}=y_{0} \in \mathbb{R},
\end{array}\right.
\end{aligned}
$$

where $r, A, B:[0, T] \rightarrow \mathbb{R}, b:[0, T] \rightarrow \mathbb{R}^{n}, \sigma:[0, T] \rightarrow$ $\mathbb{R}^{n \times n}$ are deterministic continuous functions and $T<\infty$ stands for the maturity. We assume that all of the return rate of risky asset are larger than the risk-free rate, i.e., $b^{i}(t)-$ $r(t)>0, i \in\{1,2, \cdots, n\}, t \in[0, T]$. The wealth of an investor $X_{t}$ satisfies

$$
\left\{\begin{array}{l}
\frac{d X_{t}}{X_{t}}=\sum_{i=1}^{n} \pi_{t}^{i} \frac{d S_{t}^{i}}{S_{t}^{i}}+\left(1-\sum_{i=1}^{n} \pi_{t}^{i}\right) \frac{d S_{t}^{0}}{S_{t}^{0}},\left\{\pi_{t}\right\}_{0 \leq t \leq T} \in \mathcal{A}^{\bar{\pi}}, \\
X_{0}=x_{0}=s_{0}^{0}+s_{0}^{\top} \mathbf{1}
\end{array}\right.
$$

where $\pi=\left(\pi^{1}, \cdots, \pi^{n}\right)^{\top}$ stands for the portfolio weight vector and $\mathbf{1}=(1, \cdots, 1)^{\top} \in \mathbb{R}^{n}$. A class of portfolio strategies $\mathcal{A}^{\bar{\pi}}$ is the collection of $\mathbb{R}^{n}$-valued $\mathcal{F}_{t}$-adapted process $\left\{u_{t}\right\}_{0 \leq t \leq T}$ which satisfies $\quad 0 \leq u_{t}^{i} \leq \bar{\pi}, i=1,2, \cdots, n$. The heart of the definition of the class $\mathcal{A}^{\bar{\pi}}$ is prohibition of the short selling.

We define the performance criterion $J_{t}^{\pi}$ based on the downward side of the mean square error between the liability and our wealth which improve the negative point in the previous study [1] mentioned in the introductory section:

$$
\begin{aligned}
& J_{t}^{\pi}(x, y) \\
& =\mathbb{E}\left[\frac{1}{2}\left(Y_{T}-X_{T}\right)_{+}^{2}+\frac{1}{2} \int_{t}^{T}\left(Y_{s}-X_{s}\right)_{+}^{2} d s \mid X_{t}=x, Y_{t}=y\right] .
\end{aligned}
$$

Here we use a notation $(\cdot)_{+}$s.t. $(x)_{+}=x 1_{\{x>0\}}, x \in \mathbb{R}$.

The value function of our problem $V_{t}$ is defined by

$$
V_{t}(x, y)=\inf _{\pi \in \mathcal{A}^{\bar{\pi}}} J_{t}^{\pi}(x, y),
$$

with terminal condition $V_{T}(x, y)=\frac{1}{2}(y-x)_{+}^{2}$, and the corresponding HJB equation is

$$
\partial_{t} V_{t}(x, y)+\min _{\pi \in \mathcal{A}^{\tilde{\pi}}}\left\{\mathscr{L}_{t}^{\pi} V_{t}(x, y)\right\}=0,
$$

where $\mathscr{L}_{t}^{\pi}$ is an infinitesimal generator of $\left(X_{t}, Y_{t}\right)^{\top}$ :

$$
\begin{aligned}
\mathscr{L}_{t}^{\pi} \phi(x, y) \\
=\left(r(t)+(b(t)-r(t) \mathbf{1})^{\top} \pi\right) x \partial_{x} \phi(x, y) \\
\quad+(A(t) y+B(t)) \partial_{y} \phi(x, y) \\
\quad+\frac{1}{2} x^{2} \pi^{\top} \sigma(t) \sigma(t)^{\top} \pi \partial_{x x} \phi(x, y)+\frac{1}{2}(y-x)_{+}^{2},
\end{aligned}
$$

for $\phi: \mathbb{R}^{2} \rightarrow \mathbb{R}$. We define the Hamiltonian $H$ as follow:

$$
H\left(t, x, y, \partial V_{t}, \partial^{2} V_{t}\right)=\min _{\pi \in \mathcal{A}^{\tilde{\pi}}}\left\{\mathscr{L}_{t}^{\pi} V_{t}(x, y)\right\} .
$$

\section{Numerical Method}

Under the current control set $\mathcal{A}^{\bar{\pi}}$, it is difficult to obtain the analytical solution for the HJB equation (1). Hence we employ the quadratic approximation scheme proposed by Nakano [3]. This method starts with the following time stepping

$$
V_{t_{i}}(x, y) \approx V_{t_{i+1}}+h H\left(t_{i}, x, y, \partial V_{t_{i+1}}, \partial^{2} V_{t_{i+1}}\right),
$$

and our goal is to obtain $V_{t_{i}}^{h, \bar{x}, \bar{y}}$, the approximated value function around a point $(\bar{x}, \bar{y})$ at time $t_{i}$, in the following quadratic form:

$$
\begin{aligned}
V_{t_{i}}^{h, \bar{x}, \bar{y}}(x, y)= & c_{1, i}+c_{2, i}(x-\bar{x})+c_{3, i}(y-\bar{y}) \\
& +\frac{1}{2} c_{4, i}(x-\bar{x})^{2}+c_{5, i}(x-\bar{x})(y-\bar{y}) \\
& +\frac{1}{2} c_{6, i}(y-\bar{y})^{2},
\end{aligned}
$$

where $t_{i}=i h, i=0,1, \cdots, M, h=T / M, c_{j, i} \in \mathbb{R}, j=$ $1, \cdots, 6$. Hence the key to obtain the approximate solution is the approximation of the Hamiltonian.

Before we introduce the procedure to obtain the approximate solution, we transform the Hamiltonian (2) into the form without the minimum. We assume that $\partial_{x x} V_{t}>0$, and then the Karsh-Kuhn-Tacker condition implies that

$$
H\left(t, x, y, \partial V_{t}, \partial^{2} V_{t}\right)=\mathscr{L}_{t}^{\hat{\pi}} V_{t}(x, y) .
$$

where

$$
\hat{\pi}_{i}=\min \left\{\left(\frac{-\partial_{x} V_{t}}{x \partial_{x x} V_{t}}\left(\pi_{0}\right)_{i}\right)_{+}, \bar{\pi}\right\},
$$

and $\pi_{0}(t)=\left(\sigma \sigma^{\top}\right)^{-1}(b-r \mathbf{1})(t)$.

We now show the procedure to obtain the approximate solution. Since terminal condition is given in the quadratic function of $x$ and $y$, the coefficients $\left\{c_{j, M}\right\}_{j=1, \cdots, 6}$ are determined as follows:

$$
\begin{aligned}
c_{1, M} & =\frac{1}{2}(\bar{y}-\bar{x})^{2}, \quad c_{2, M}=-(\bar{y}-\bar{x}), \quad c_{3, M}=\bar{y}-\bar{x} \\
c_{4, M} & =1, c_{5, M}=-1, c_{6, M}=1 .
\end{aligned}
$$

Next we define $q \in \mathbb{R}^{2}$ and $P \in \mathbb{R}^{2 \times 2}$ as follows:

$$
q=\left(c_{2, M}, c_{3, M}\right)^{\top}, \quad P=\left(\begin{array}{ll}
c_{4, M} & c_{5, M} \\
c_{5, M} & c_{6, M}
\end{array}\right),
$$


and then we find that $\partial V_{t_{M}}=P(x-\bar{x}, y-\bar{y})^{\top}+q$ and $\partial^{2} V_{t_{M}}=P$. Applying the regression method, we approximate the Hamiltonian by the quadratic function:

$$
\begin{aligned}
H & \left(t_{n-1}, x, y, P(x-\bar{x}, y-\bar{y})^{\top}+q, P\right) \\
\approx & L_{1}+L_{2}(x-\bar{x})+L_{3}(y-\bar{y}) \\
& +\frac{1}{2} L_{4}(x-\bar{x})^{2}+L_{5}(x-\bar{x})(y-\bar{y}) \\
& +\frac{1}{2} L_{6}(y-\bar{y})^{2},
\end{aligned}
$$

where $L_{j} \in \mathbb{R}, j=1, \cdots, 6$. To determine the coefficients $\left\{L_{j}\right\}_{j=1, \cdots, 6}$, we employ the least square method with $K$ sample points $\left(x_{k}, y_{k}\right), k=1, \cdots, K$ which are randomly chosen from the circle area with radius $r$ and the central point $(\bar{x}, \bar{y})$. By (3) and (6), we have

$$
c_{j, M-1}=c_{j, M}+h L_{j}, \quad j=1, \cdots, 6 .
$$

Replacing $M-1$ into $M$, going back to equation (5) and repeating this procedure, we obtain the coefficients $\left\{c_{j, i}\right\}_{j=1, \cdots, 6}$ which is equivalent to find the approximated value function $V_{t_{i}}^{h, \bar{x}, \bar{y}}$. We are able to find the optimal strategy substitute $V_{t_{i}}^{h, \bar{x}, \bar{y}}$ into the equation (4).

We remark that the above procedure to obtain the optimal strategy does not contain processes which need a high computational cost. This is the advantage employing the quadratic approximation method. Furthermore, if we only impose the no-short-selling constraint, i.e., $\bar{\pi}=\infty$, we find the explicit form of $\hat{\pi}$,

$\hat{\pi}=\frac{\left(-\left(c_{2, i}+c_{4, i}(x-\bar{x})+c_{5, i}(y-\bar{y})\right)\right)_{+}}{x c_{4, i}} \pi_{0} \mathbf{1}_{\{(\bar{x}<\bar{y}) \wedge(x<y)\}}$,

and the explicit recursive formula for $\left\{c_{j, i}\right\}_{j=1, \cdots, 6}$ :

$$
\begin{aligned}
& c_{1, i}=c_{1, i+1}+h\left\{r\left(t_{i}\right) \bar{x} c_{2, i+1}+\left(\bar{y} A\left(t_{i}\right)+B\left(t_{i}\right)\right) c_{3, i+1}\right. \\
& \left.-\left|\sigma^{\top} \pi_{0}\right|^{2}\left(t_{i}\right) \frac{c_{2, i+1}^{2}}{2 c_{4, i+1}} \mathbf{1}_{\left\{c_{2, i+1} \leq 0\right\}}+\frac{1}{2}(\bar{y}-\bar{x})^{2}\right\}, \\
& c_{2, i}=c_{2, i+1}+h\left\{r\left(t_{i}\right) c_{2, i+1}+r\left(t_{i}\right) \bar{x} c_{4, i+1}\right. \\
& +\left(\bar{y} A\left(t_{i}\right)+B\left(t_{i}\right)\right) c_{5, i+1} \\
& \left.-\left|\sigma^{\top} \pi_{0}\right|^{2}\left(t_{i}\right) c_{2, i+1} \mathbf{1}_{\left\{c_{2, i+1} \leq 0\right\}}-(\bar{y}-\bar{x})\right\}, \\
& c_{3, i}=c_{3, i+1}+h\left\{r\left(t_{i}\right) \bar{x} c_{5, i+1}+A\left(t_{i}\right) c_{3, i+1}\right. \\
& +\left(\bar{y} A\left(t_{i}\right)+B\left(t_{i}\right)\right) c_{6, i+1} \\
& \left.-\left|\sigma^{\top} \pi_{0}\right|^{2}\left(t_{i}\right) \frac{c_{2, i+1} c_{5, i+1}}{c_{4, i+1}} \mathbf{1}_{\left\{c_{2, i+1} \leq 0\right\}}+\bar{y}-\bar{x}\right\}, \\
& c_{4, i}=c_{4, i+1}+h\left\{2 r\left(t_{i}\right)-\left|\sigma^{\top} \pi_{0}\right|^{2}\left(t_{i}\right) c_{4, i+1} \mathbf{1}_{\left\{c_{2, i+1} \leq 0\right\}}+1\right\}, \\
& c_{5, i}=c_{5, i+1}+h\left\{r\left(t_{i}\right)+A\left(t_{i}\right)-1\right. \\
& \left.-\left|\sigma^{\top} \pi_{0}\right|^{2}\left(t_{i}\right) c_{5, i+1} \mathbf{1}_{\left\{c_{2, i+1} \leq 0\right\}}\right\}, \\
& c_{6, i}=c_{6, i+1}+h\left\{2 A\left(t_{i}\right) c_{6, i+1}+1\right. \\
& \left.-\left|\sigma^{\top} \pi_{0}\right|^{2}\left(t_{i}\right) \frac{c_{5, i+1}^{2}}{c_{4, i+1}} \mathbf{1}_{\left\{c_{2, i+1} \leq 0\right\}}\right\},
\end{aligned}
$$

under the condition $c_{4, i}>0, i=1, \cdots, n$. Therefore we are able to obtain a proxy of the optimal strategy with remarkable high speed.

\section{Numerical Results}

In this section, we serve numerical simulations base on our method using the empirical data provided by the Japanese organizations. The usage of data is the same as in the literature [1]. Let $C_{t}$ and $B_{t}$ be the estimated income and expense of the pension fund which are determined by the data published by the Japanese Ministry of Health, Labour and Welfare [4]. We set $t=0$ as the year 2040 when the estimated shortfall of the pension fund starts to expand drastically (see Figure 1). We set $A(t)=0$ and $B(t)$ as the numerical differentiation fo $B_{t}-C_{t}$ and then we regard the estimated shortfall of the pension fund as the liability.

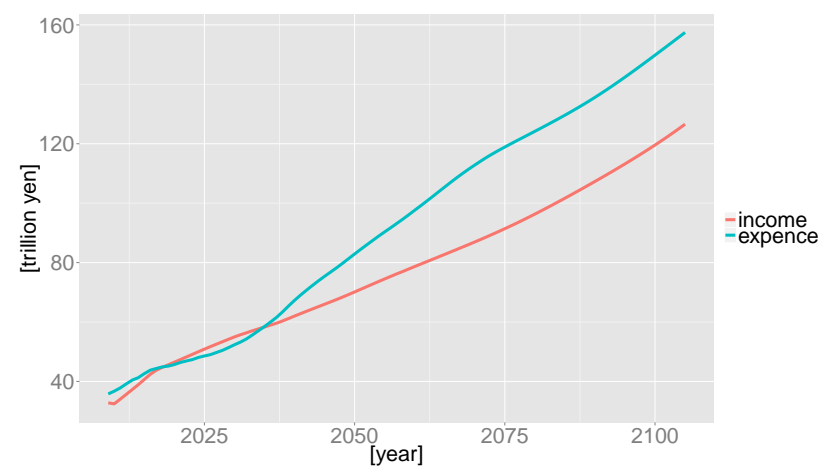

Fig. 1: Estimations of the income and expense of the Japanese welfare pensions. Red and blue lines indicate $C_{t}$, the estimated income, and $B_{t}$, the estimated expense respectively.

We next determine the risk-free rate and the expected return rates and volatilities of risky assets. We invest the following four assets: indices of the domestic bond, the domestic stock, the foreign bond and the foreign stock; we set $n=4$ and number them sequentially. According to the estimations of return rate and volatilities by the Government Pension Investment Fund, Japan [5], we construct $b(t)$ and $\sigma_{S}(t)$ as follows: $b^{1}(t)=3 \%, b^{2}(t)=4.8 \%, b^{3}(t)=3.5 \%$ and $b^{4}(t)=5.0 \% ; \sigma(t)$ is the Cholesky decomposition of the following variance-covariance matrix of the assets:

$$
\Sigma=\left(\begin{array}{cccc}
29.7 & 18.2 & -4.39 & -5.41 \\
18.2 & 495 & -77.8 & 119 \\
-4.39 & -77.8 & 181 & 147 \\
-5.41 & 119 & 147 & 394
\end{array}\right) \times 10^{-4}
$$

We choose a money market account as the risk-free asset and we set $r(t)=0.0 \%$.

In the following two subsections, we consider the cases: (i) the no-short-selling constraint; (ii) the upper bound constraint for the portfolio weight. We calculate the optimal portfolio weight by the method mentioned in Section 3 
and run investment simulations using the Euler-Maruyama scheme. We set the related parameters as in the Table 1 .

\begin{tabular}{|c|c|c|}
\hline Symbol & Description & Value \\
\hline$T$ & terminal time & 15 \\
\hline$h$ & $\begin{array}{l}\text { time stepping for calculation } \\
\text { of optimal strategy }\end{array}$ & 0.001 \\
\hline$N$ & number of sample paths & 1000 \\
\hline$\Delta t$ & $\begin{array}{l}\text { time stepping for simulation } \\
\quad \text { (quarterly rebalance) }\end{array}$ & 0.25 \\
\hline$r$ & radius of the regression region & $\sqrt{2 h}$ \\
\hline$K$ & $\begin{array}{l}\text { number of sample points } \\
\text { for regression }\end{array}$ & 10 \\
\hline
\end{tabular}

Table 1: Parameters

Let us introduce the mean hedging error

$$
E_{t}=\sum_{i=1}^{N} \frac{\left(Y_{t}-X_{t}^{i}\right)_{+}}{N}
$$

to discuss the performance of the strategy, where $X_{t}^{i}$ is the $i$-th sample path of the investment simulation.

\subsection{Case of no-short-selling constraint}

In this subsection, let us consider the case of no-short selling constraint only, i.e., $\bar{\pi}=\infty$.

Figure 2 displays a sample path of a investment simulation. We find that our optimal portfolio is mainly constructed by the domestic bond, the most low-risk asset. The foreign bond is the second weighted asset and it is included in the optimal portfolio with a considerable weight. The weight of domestic and foreign stocks are little and thus the high-risk assets do not play an important role in our hedging strategy. These facts imply that the optimal hedging method for the liability is borrowing the money a lot and investing the bonds, the low-risk assets. Since there does not exist the limit for the amount of money borrowing, we are able to choose the strategy that investing the low-risk assets a lot and earning the desired profit with low-risk.

Another feature of our optimal strategy is that the large hedging error occurred in earlier stage of investment is quickly hedged. In Figure 2, we observe that the portfolio weights near the start and terminal times are different despite the tracking errors are not much different. It is quite reasonable behavior: the tracking error which is occurred in earlier time and is not hedged expands by the reinvestment effect, and thus we need to cover it quickly.

Figure 3 describes the statistical results of the investment simulation. We observe that the mean wealth indicated by the blue line tracks the liability indicated by the red line well. The mean hedging error $E_{t}$ described in the lower panel of Figure 3 is approximately $4.5 \%$ of the liability and it is the equivalent level without the no-short-selling constraint studied in [1]. Hence we find that the liability tracking ability of our optimal portfolio strategy does not drop if we restrict the short selling.

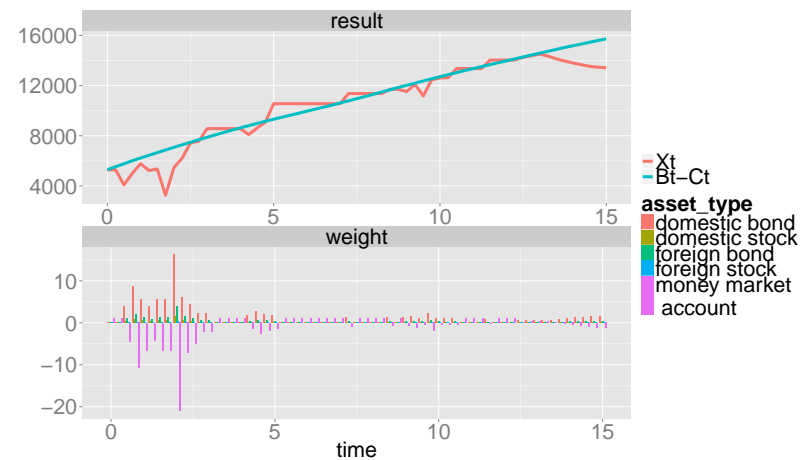

Fig. 2: A sample of the investment simulation in the case of $\bar{\pi}=\infty$. Red and blue lines in the upper panel indicate the time evolution of $X_{t}$, our wealth, and $B_{t}-C_{t}$, the liability, respectively. Red, yellow, green, blue and purple bars in the lower panel display the portfolio weights of the domestic bond, the domestic stock, the foreign bond, the foreign stock and money market account respectively.

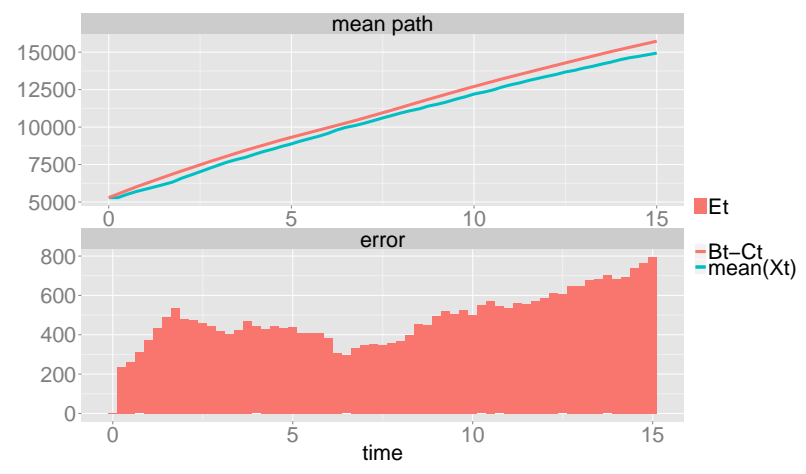

Fig. 3: Statistical results of the investment simulation in the case of $\bar{\pi}=\infty$. Red and blue lines in the upper panel represent the time evolution of mean $\left(X_{t}\right)=\sum_{i=1}^{N} \frac{X_{t}^{i}}{N}$, the mean wealth, and $B_{t}-C_{t}$, the liability, respectively. Red bar in the lower panel indicate the time evolution $E_{t}$, the mean hedging error.

\subsection{Cases of upper bound constraint for the portfolio weights}

The optimal portfolio investigated in the previous subsection is hard to apply the real business since the large money borrowing is quite difficult to realize. Therefore we study the case that we impose the upper bound for the portfolio weights. We set $\bar{\pi}=1$, i.e., the weight of each asset is bounded by $100 \%$ and hence the minimum weight of the money market account is $-300 \%$. Although the bound of the money borrowing is not little, results of investment simulations imply that the pension fund faces the severe situation under the current restriction.

Figure 4 displays a sample path investment simulation which is a case that the liability is well hedged. We find that the portfolio weights are not concentrated on the domestic bond: all of the weights closes up the upper bound around $t=1$. We also note that the weight rises up in the increasing 
order of the risk, i.e, the domestic bond is the most weighted asset, the foreign bond is second one, and the domestic and foreign stocks follows sequentially.

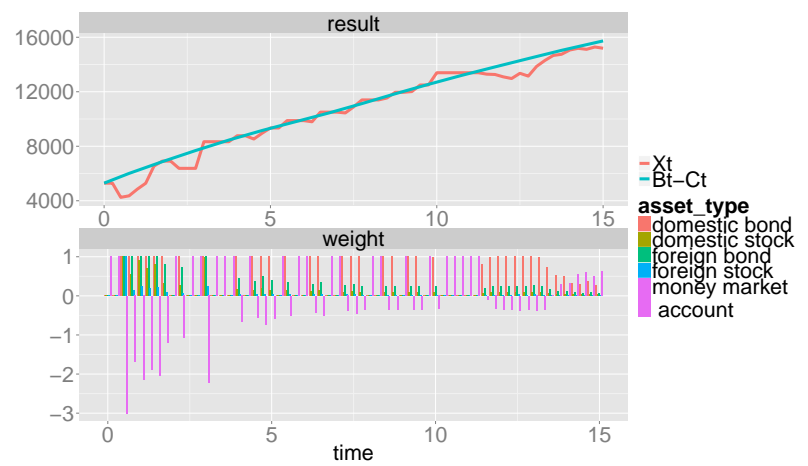

Fig. 4: A sample of the investment simulation under the constraint $\bar{\pi}=1$ : the well hedged case. Red and blue lines in the upper panel indicate the time evolution of $X_{t}$, our wealth, and $B_{t}-C_{t}$, the liability, respectively. Red, yellow, green, blue and purple bars in the lower panel display the portfolio weights of the domestic bond, the domestic stock, the foreign bond, the foreign stock and money market account respectively.

Under the constraint $\bar{\pi}=1$, the poor hedged results as shown in Figure 5 appear frequently, though they are rarely found in the case of $\bar{\pi}=\infty$. Figure 6 implies that the result displayed in Figure 5 is not a special case. The mean hedging error $E_{t}$ described in the lower panel of Figure 6 is approximately $21 \%$ of the liability and which is much higher than $4.5 \%$ studied in the previous subsection. Almost all of the portfolio weights in Figure 5 reach the upper limit however the profit earned by those assets is not enough to recover the tracking error.

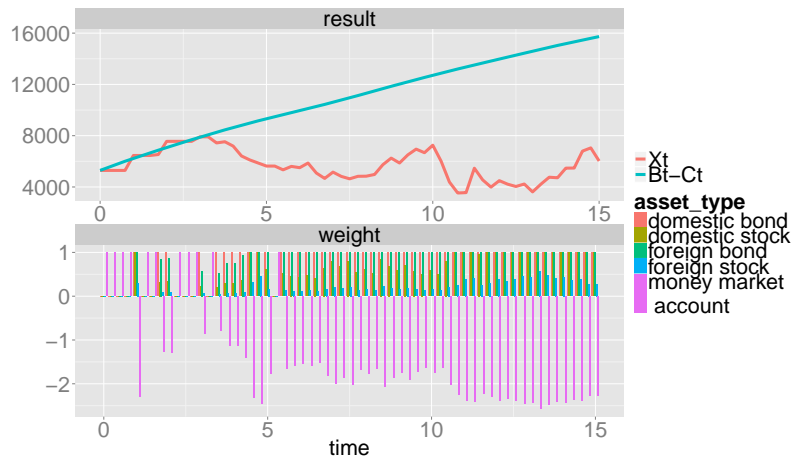

Fig. 5: A sample of the investment simulation under the constraint $\bar{\pi}=1$ : the poor hedged case. Red and blue lines in the upper panel indicate the time evolution of $X_{t}$, our wealth, and $B_{t}-C_{t}$, the liability, respectively. Red, yellow, green, blue and purple bars in the lower panel display the portfolio weights of the domestic bond, the domestic stock, the foreign bond, the foreign stock and money market account respectively.
Let us discuss the implication of these results. Our simulations imply the imbalance between the growth rate of the liability and the profitability of the assets. We denote by $\alpha$ the growth rate of the liability and it is $7.6 \%$ which is higher than all of $b^{i}$. Since our purpose is minimising the downside tracking error, it is not enough that the expected return rates of the assets close to $\alpha$ : the high volatility assets possibly disturb the tracking. Furthermore the current restriction which arrows the $300 \%$ money borrowing is even weak for the practice. Therefore the implication is summarized that the imbalance of the growth rate of the liability and the profitability of the invested assets should be resolved.

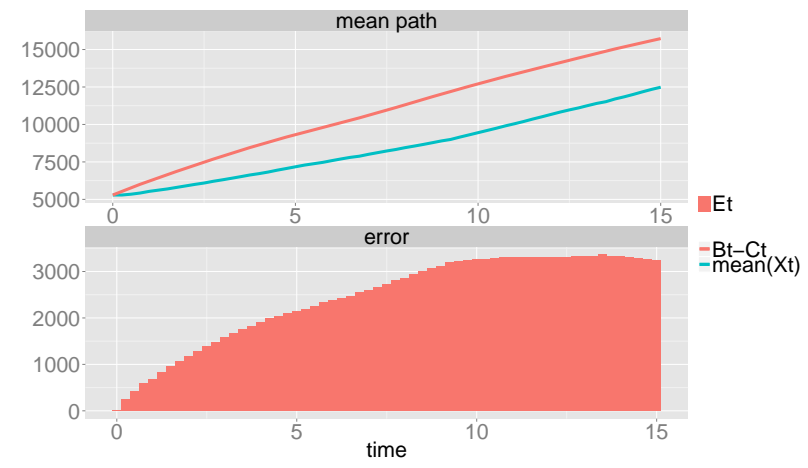

Fig. 6: Statistical results of the investment simulation in the case of $\bar{\pi}=1$. Red and blue lines in the upper panel represent the time evolution of mean $\left(X_{t}\right)=\sum_{i=1}^{N} \frac{X_{t}^{i}}{N}$, the mean wealth, and $B_{t}-C_{t}$, the liability, respectively. Red bar in the lower panel indicate the time evolution $E_{t}$, the mean hedging error.

\section{Summary}

In the present paper, we develop the portfolio strategy from the strategy proposed by Ieda et. al. [1]. The previous study remains two points which should be improved: (i) penalising the wealth of the investor exceed the liability; (ii) permitting the large short selling. To resolve these points, we employ a lower mean square error from the tracked liability as the performance criterion and restrict the portfolio weight to a positive and bounded value. In this case we are not able to obtain an analytical solution and thus we employ the numerical scheme proposed by Nakano [3] to solve the corresponding HJB equation.

We run numerical simulations using the empirical data provided by Japanese organizations under the two types of constraints: (i) the no-short-selling constraint; (ii) the upper bound constraint for the portfolio weights. The implications obtained by these simulations are summarized as follows. We find that the liability tracking ability of our optimal portfolio strategy does not drop if we restrict the short selling. The results studied in Section 4.1 indicates that the mean hedging error is approximately $4.5 \%$ of the liability and it is the equivalent level without the no-short-selling constraint studied in [1]. Under the no-short-selling constraint, the hedge is realized by borrowing the money a lot and investing 
the low-risk assets. According to this result, we restrict the large money borrowing by imposing the upper bound constraint for the portfolio weights in Section 4.2. Then we find a number of sample paths which have a large tracking error. It implies that the imbalance between the growth rate of the liability and the profitability of the assets.

\section{Acknowledgements}

This work is supported by a collaboration research project with the Government Pension Investment Fund in Japan in 2013-2014.

\section{References}

[1] Masashi Ieda, Takashi Yamashita, and Yumiharu Nakano. A Liability Tracking Approach to Long Term Management of Pension Funds. Journal of Mathematical Finance, 03(03):392-400, 2013.

[2] Harold J Kushner and Paul G Dupuis. Numerical methods for stochastic control problems in continuous time, volume 24. Springer, 2000.

[3] Yumiharu Nakano. A quadratic approximation scheme for Hamilton-Jacobi-Bellman equations. Working Paper, Tokyo Institute of Technology, Graduate School of Innovation Management, pages 1-23, 2013.

[4] The Japanese Ministry of Health, Labour and Welfare. http://www.mhlw.go.jp/seisakunitsuite/ bunya/nenkin/nenkin/zaisei-kensyo/index . html [accessed 01 October 2014].

[5] The Government Pension Investment Fund in Japan. http://www.gpif.go.jp/operation/committee/ pdf/h191001_appendix_05.pdf [accessed 01 October 2014]. 